\title{
Human Milk Phthalate Diester Levels in Turkey
}

Meltem Dinleyici*

Department of Social Pediatrics, Eskisehir Osmangazi University, Eskisehir, Turkey

*Corresponding author: Dinleyici M, Department of Social Pediatrics, Eskisehir Osmangazi University, Eskisehir, Turkey, Tel: +9022223929279/2708; E-mail: meltemayata@hotmail.com

Received date: September 11, 2018; Accepted date: September 27, 2018; Published date: October 02, 2018

Copyright: $@ 2018$ Dinleyici M. This is an open-access article distributed under the terms of the Creative Commons Attribution License, which permits unrestricted use, distribution, and reproduction in any medium, provided the original author and source are credited.

\begin{abstract}
Introduction: Newborn and infants are among the most susceptible age groups to endocrine disruption due to potential environmental toxins like phthalates. Differing results have been found on phthalate exposure through human milk from different geographic parts of the world. In this study, we aim to evaluate six different phthalate diesters in human milk samples.

Methods: In this part of the Human Milk Artificial Pollutants (HUMAP) study, human milk samples were analyzed using GC-MS to indicate the presence of bis (2-ethylhexyl) phthalate (DEHP), benzyl butyl phthalate (BBP), dibutyl phthalate (DBP), di-“isononyl" phthalate (DINP), di-"isodecyl" phthalate (DIDP), and di-n-octyl phthalate (DNOP).

Results: This study included 72 mothers aged 18 to 41 years; human milk samples were taken from the mothers who gave their consent to participate within 7 to 79 days after birth (mean $34 \pm 20$ days). We did not detected phthalate esters as DEHP, BBP, DBP, DINP, DIDP, and DNOP in 72 human milk samples.

Discussion: In this study, we did not find phthalate diesters in human milk samples in Turkey. Different results of previous studies about human milk phthalate levels might be related to geography, maternal factors, or analytical methods. In conclusion, potential further studies should continue for potential chemical contamination of human milk.
\end{abstract}

Keywords: Human milk; Phthalate; Phthalate diesters; Breastfeeding

\section{Introduction}

Phthalates have been widely used in various fields, including food, cosmetics, and pharmaceuticals. Phthalates are found ubiquitously in the environment. They migrate easily and penetrate various every day products and reach organisms via skin contact, food or air [1,2]. Phthalates and their metabolites are widely detected in urine, serum, human milk, amniotic fluid, umbilical cord blood, semen, and saliva [1-3]. There is widespread risk of exposure to phthalates during pregnancy because of their ability to cross the placenta, which may adversely affect fetal outcomes following in-utero exposure $[3,4]$. Exposures of phthalates during prenatal and postnatal development possibly resulting in reproductive disorders as antiandrogenic and/or proestrogenic effects in humans [2,5-7]. Phthalates may also play a role in obesity [2].

Human milk phthalate levels vary by geographical region and maternal factors including cosmetics and food preferences [8]. Infants are at increased risk, as they are exposed early in life to several different sources of phthalates due to a higher prevalence of exposure among women during pregnancy and lactation [9]. To the best of our knowledge, there are no studies on human milk phthalate levels in Turkey. In this study, we will evaluate six different phthalate monoesters and phthalate diesters in human milk samples.

\section{Materials and Methods}

The Human Milk Artificial Pollutants (HUMAP) study is a crosssectional study designed to evaluate the presence of mycotoxins, bisphenol A, phthalates, and antibiotic residues in human milk samples in Turkey. In this part of the HUMAP study, we evaluated the presence of phthalate levels in human milk samples. The project was approved by the Eskisehir Osmangazi University Local Ethics Committee, and all participants gave written informed consent. The study was supported by the Eskisehir Osmangazi University Scientific Research Grant.

We evaluated all lactating mothers with 7- to 90-day-old babies at the Eskisehir Osmangazi University and Eskisehir Maternity and Children Hospital, Eskisehir, Turkey. Maternal age, number of pregnancies, gestational age, and number of breastfeeding days were noted for each study participant. Within the scope of the study and after receiving informed consent, 3-5 cc of human milk was obtained from each participant under sterilized conditions and stored at $-20^{\circ} \mathrm{C}$ until the end of the study period. Phthalate levels were evaluated with the in-house gas chromatography/mass spectroscopy (GC/MS) technique. We evaluated six different phthalate monoesters and phthalate diesters: Bis (2-ethylhexyl) phthalate (DEHP), benzyl butyl phthalate (BBP), dibutyl phthalate (DBP), di-“isononyl" phthalate (DINP), di-"isodecyl" phthalate (DIDP), and di-n-octyl phthalate (DNOP). All analyses were performed in the same reference laboratories. The results are shown in parts per million (ppm), and the detection limit is less than $20 \mathrm{ppm}$.

A statistical analysis was performed using the SPSS package program, version 11.5 (Chicago, Illinois, United States). Values that are normally distributed are expressed as mean and standard deviation. The Mann-Whitney $U$ test was used to compare unpaired data among the groups. A p-value of less than 0.05 was considered statistically significant. 


\section{Results}

This study included 72 mothers aged 18 to 41 years $(29.9 \pm 5.97$ years). Human milk samples were taken from mothers who gave consent to participate within 7 to 79 days after birth (mean $34 \pm 20$ days). 38 mothers had cesarean deliveries (52.8\%) and 34 had spontaneous vaginal deliveries $(47.2 \%)$. None of the mothers took antibiotics during pregnancy. 25 mothers $(34.7 \%)$ were on antibiotics during delivery and 41 mothers were on antibiotics postpartum (56.9\%). The average gestational period was $38.6 \pm 1.23$ weeks $(37-42$ weeks) and the average birth weight was $3470 \pm 551 \mathrm{~g}$ (2065-5270 g).

We did not detected phthalate esters as DEHP, BBP, DBP, DINP, DIDP, and DNOP in 72 human milk samples with GC/MS technique, all samples were below the detection limit.

\section{Discussion}

In this study, we did not find six different phthalate diesters in 72 human milk samples in Turkey. Previous studies from other geographical locations showed different results [8,10-13]. The differences between the studies might be related to geography, maternal factors, or analysis methods. Hagberg, et al. [9] evaluated human milk phthalate levels with GC-MS in 42 mothers in Sweden in 2001 14-20 days after birth. The concentration of phthalates and phthalate metabolites in the milk was close to or below the limit of detection, except for one sample with a high DEHP concentration. In 2004, Calafat, et al. [13] showed several phthalate metabolites in human milk samples, suggesting that phthalates transferred to the infants via breastfeeding. Kim, et al. [14] evaluated six phthalate metabolites concentrations in 62 human milk samples received one month postpartum in Korea; monoethyl phthalate (MEP) was detected in $100 \%$ the samples, and other phthalate monoesters were detected in $79 \%-89 \%$ of the samples. They found that up to $8 \%$ of infants exceeded the reference dose of anti-androgenicity for DEHP and 6\% of infants exceeded the tolerable daily intake for DnBP. These phthalate monoesters in human milk showed significantly associated with maternal consumption of whipped cream or purified water. Latini et al. [8] evaluated the human milk phthalate metabolites from 62 healthy mothers living in Italy with a LC/LC-MS/MS method. They found that mono-isobutyl phthalate and mono(2-ethylhexyl) phthalate were present in all milk samples, mono-n-butyl phthalate was present in $64.5 \%$, and mono-benzyl phthalate was found in $43.5 \%$ of samples. Fromme, et al. [15] evaluated 15 phthalates and their metabolites in 78 human milk samples in Germany; the aforementioned average/high intake values corresponded to only about $2 \%$ and $7 \%$, respectively, and they concluded that exposure to phthalates from human milk poses any significant health risk for infants. Hines, et al. [16] detected oxidative monoester phthalate metabolites in $10 \%$ of the human milk samples of 33 lactating women in the United States. Lin, et al. [17] studied phthalate metabolites in pregnant women and their newborns in Taiwan and showed that phthalate metabolite levels were low in human milk and cord blood samples comparing to urinary levels. According to their study, among breast-fed infants, the mean daily intake during the first six months was estimated at $167 \mathrm{microg} / \mathrm{dl}$ for DEHP and $<1 \mathrm{microg} / \mathrm{dl}$ for DBP and DEP. Polinski, et al. [4] evaluated 15 phthalate metabolites in the urine of 446 pregnant women; the phthalate metabolites with the highest concentrations were monoethyl phthalate and monocarboxyisooctyl phthalate and showed correlations between human milk phthalate levels and the mother's age, obesity. A study in Denmark and Finland, reported higher concentrations of some phthalate metabolites in human milk, while low levels have been reported in Sweden despite similarities in lifestyle and demographics in Sweden, Denmark, and Finland $[11,12,18]$.

Absence of phthalates in our study samples might be related to maternal food preferences and study location. We did not perform a phthalate analysis on maternal urine samples, and it is difficult to properly address the low exposure to phthalates without this data. Hines, et al. [16] and Hogberg, et al. [9] suggested that phthalate metabolites are most frequently detected in the urine of lactating women and are less often detected in human milk, as urinary phthalate concentrations reflect maternal exposure and do not represent the concentrations of oxidative metabolites in other body fluids, especially human milk. Zhu, et al. [19] demonstrated fluctuated human milk phthalate esters during their follow period over a sixmonth postpartum period. They concluded that multiple sample collections would provide a better estimate of the exposure to phthalates in human milk than a single sample among breast-fed infants.

Food constitutes the most prevalent source of human exposure to phthalates, and human milk phthalate levels might be related to maternal food preferences [20]. Beltifa, et al. [21] evaluated phthalate monoesters and diesters in packaged foods (grain and grain products, milk and dairy products, fats and oils, beverages, fish, and sweets) in Tunisia with GC/MS and found that benzyl butyl phthalate (BBP) was highly concentrated in puff pastry, milk, butter, yogurt, oil, water, candy, fruit juices, and chocolate. Sidlowska et al. [20] evaluated eight phthalate metabolites in 107 children 10-12 years of age in Slovakia and found a statistically significant association between some phthalate metabolites and the consumption of milk, dairy products, margarine, hot beverages, and baguettes as well as the use of cosmetic products, nail polish, and body lotion. Various phthalates (most commonly DEHP, DnBP, and DiBP) have been detected in food products such as milk, fat-enriched foods, meat, fish, seafood, beverages, grains, and vegetables [10]. Ji, et al. [22] evaluated 448 samples including foodstuffs, ambient PM10, drinking water, soil, and indoor dust samples in China to determine the distribution of six priority phthalates. They highlighted that environmental factors might be related to exposure to phthalates as well as food preference. Pregnancy creates a critical risk of phthalate exposure for mothers due to their nutritional preferences, cosmetics, and environmental factors.

Phthalate exposure has been found to be associated with endocrine disruption, respiratory effects, and reproductive and developmental toxicity [17]. De Cock, et al. [23] found that exposure to phthalates was associated with body mass index as well as with head circumferences during the first year after birth. Main, et al. [11] investigated whether phthalate monoester contamination of human breast milk had any influence on the postnatal surge of reproductive hormones in newborn boys as a sign of testicular dysgenesis, and they found that phthalate exposure in newborn boys affected leydig cell development and function.

\section{Conclusion}

We have some limitations for this study. We evaluated only human milk samples for phthalate levels, and we did not perform analysis in other maternal and/or infant body fluids, especially in maternal urine. We also collected human milk samples from mothers living in same city, this would be advantage for eliminating potential different results in same country, however further multicenter studies would help to evaluate the differences at country-based level. 
Biomonitoring is a very useful tool for assessing human exposure to environmental pollutants. In this study, we did not detect phthalate diesters in human milk samples. Considering our previous studies on environmental pollutants and drugs in human milk samples, these results are different than we expected. We previously found antibiotic residues in human milk from mothers who had not received antibiotics during pregnancy [24]. We detected significantly higher levels of ochratoxin A, deoxynivalenol, and zearalenone levels in human milk, but we did not detect aflatoxin M1 levels above the threshold levels $[25,26]$. These results and others from different geographical areas showed the need for analyses of complex mixtures to obtain more information on inter-individual and temporal variability of human exposure to different types of chemicals [27].

Breastfeeding is considered a gold-standard for nutrition and its recommend for all infants [28]. Instead, comprehensive programs should be developed to regularly investigate and control toxins in both human and animal food chains so that the amount of toxins can be reduced and their side effects can be prevented. In conclusion, further studies should continue to evaluate human milk for potential environmental pollutants.

\section{References}

1. Sicinska P (2018) Di-n-butyl phthalate, butylbenzyl phthalate and their metabolites induce haemolysis and eryptosis in human erythrocytes. Chemosphere 203: 44-53.

2. Martinez-Arguelles DB, Campioli E, Culty M, Zirkin BR, Papadopoulos V (2013) Fetal origin of endocrine dysfunction in the adult: The phthalate model. J Steroid Biochem Mol Biol 137: 5-17.

3. Minatoya M, Araki A, Miyashita C, Ait Bamai Y, Itoh S, et al. (2018) Association between prenatal bisphenol $\mathrm{A}$ and phthalate exposures and fetal metabolic related biomarkers: The hokkaido study on environment and children's health. Environ Res 161: 505-511.

4. Polinski KJ, Dabelea D, Hamman RF, Adgate JL, Calafat AM, et al. (2018) Distribution and predictors of urinary concentrations of phthalate metabolites and phenols among pregnant women in the Healthy Start Study. Environ Res 162: 308-317.

5. Wang X, Sheng N, Cui R, Zhang H, Wang J, Dai J (2017) Gestational and lactational exposure to di-isobutyl phthalate via diet in maternal mice decreases testosterone levels in male offspring. Chemosphere 172: 260-267.

6. Andrade AJ, Grande SW, Talsness CE, Grote K, Golombiewski A, et al. A dose-response study following in utero and lactational exposure to di-(2 ethylhexyl) phthalate (DEHP): Effects on androgenic status, developmental landmarks and testicular histology in male offspring rats. Toxicology 225: 64-74.

7. Hauser R, Meeker JD, Singh NP, Silva MJ, Ryan L, et al. (2007) DNA damage in human sperm is related to urinary levels of phthalate monoester and oxidative metabolites. Hum Reprod 22: 688-695.

8. Latini G, Wittassek M, Del Vecchio A, Presta G, De Felice C, et al. (2009) Lactational exposure to phthalates in Southern Italy. Environ Int 35: 236-239.

9. Hogberg J, Hanberg A, Berglund M, Skerfving S, Remberger M, et al. (2008) Phthalate diesters and their metabolites in human breast milk, blood or serum, and urine as biomarkers of exposure in vulnerable populations. Environ Health Perspect 116: 334-339.

10. Kappenstein O, Vieth B, Luch A, Pfaff K (2012) Toxicologically relevant phthalates in food. EXS 101: 87-106.

11. Main KM, Mortensen GK, Kaleva MM, Boisen KA, Damgaard IN, et al. (2006) Human breast milk contamination with phthalates and alterations of endogenous reproductive hormones in infants three months of age. Environ Health Perspect 114: 270-276.

12. Mortensen GK, Main KM, Andersson AM, Leffers H, Skakkebaek NE (2005) Determination of phthalate monoesters in human milk, consumer milk, and infant formula by tandem mass spectrometry (LC-MS-MS) Anal Bioanal Chem 382: 1084-1092.

13. Calafat AM, Slakman AR, Silva MJ, Herbert AR, Needham LL (2004) Automated solid phase extraction and quantitative analysis of human milk for 13 phthalate metabolites. J Chromatogr B Analyt Technol Biomed Life Sci 805: 49-56.

14. Kim S, Lee J, Park J, Kim HJ, Cho G, et al. (2015) Concentrations of phthalate metabolites in breast milk in Korea: estimating exposure to phthalates and potential risks among breast-fed infants. Sci Total Environ 508: 13-19.

15. Fromme H, Gruber L, Seckin E, Raab U, Zimmermann S, et al. (2011) Phthalates and their metabolites in breast milk--results from the Bavarian Monitoring of Breast Milk (BAMBI). Environ Int 37: 715-722.

16. Hines EP, Calafat AM, Silva MJ, Mendola P, Fenton SE (2009) Concentrations of phthalate metabolites in milk, urine, saliva, and Serum of lactating North Carolina women. Environ Health Perspect 117: 86-92.

17. Lin S, Ku HY, Su PH, Chen JW, Huang PC (2011) Phthalate exposure in pregnant women and their children in central Taiwan. Chemosphere 82: 947-955.

18. Frederiksen H, Skakkebaek NE, Andersson AM (2007) Metabolism of phthalates in humans. Mol Nutr Food Res 51: 899-911.

19. Zhu J, Phillips SP, Feng YL, Yang X (2006) Phthalate esters in human milk: Concentration variations over a 6 -month postpartum time. Environ Sci Technol 40: 5276-5281.

20. Sidlovska M, Petrovicova I, Kolena B, Pilka T, Sovcikova E, Trnovec T (2017) Exposure of children to phthalates and the impact of consumer practices in Slovakia. Rev Environ Health 32: 211-214.

21. Beltifa A, Feriani A, Machreki M, Ghorbel A, Ghazouani L, et al. (2017) Plasticizers and bisphenol A, in packaged foods sold in the Tunisian markets: Study of their acute in vivo toxicity and their environmental fate. Environ Sci Pollut Res Int 24: 22382-22392.

22. Ji Y, Wang F, Zhang L, Shan C, Bai Z, et al. (2014) A comprehensive assessment of human exposure to phthalates from environmental media and food in Tianjin, China. J Hazard Mater 279: 133-140.

23. de Cock M, de Boer MR, Lamoree M, Legler J, van de Bor M (2014) First year growth in relation to prenatal exposure to endocrine disruptors-A Dutch prospective cohort study. Int J Environ Res Public Health 11: 7001-7021.

24. Dinleyici M, Kilic Yildirim G, Aydemir G, Barsan Kaya T, Bildirici Y, et al. (2017). Screening of 30 antibiotic residues in human milk with multiresidue method based on chip technology. Pedia, Cogent Med 4: 1408251.

25. Dinleyici M, Kilic-Yildirim G, Aydemir O, Barsan-Kaya T, Carman KB (2018) Human milk Ochratoxin A levels and possible relationship with maternal dietary habits in Turkey. 5th International Conference on Nutrition and Growth, Paris, France.

26. Dinleyici M, Kilic-Yildirim G, Aydemir O, Barsan-Kaya T, Carman KB (2018) Human milk aflatoxin M1 (AFM1) levels with and relationship with maternal dietary habits. Osmangazi J Med 40: 86-91.

27. Schlumpf M, Kypke K, Wittassek M, Angerer J, Mascher H, et al. (2010) Exposure patterns of UV filters, fragrances, parabens, phthalates, organochlor pesticides, PBDEs, and PCBs in human milk: Correlation of UV filters with use of cosmetics. Chemosphere 81: 1171-1183.

28. Dieterich CM, Felice JP, O'Sullivan E, Rasmussen KM (2013) Breastfeeding and health outcomes for the mother-infant Dyad. Pediatr Clin North Am 60: 31-48. 